\title{
Current Moments at ELF of Lightning Discharges Associated with a Mature Tropical Hurricane
}

\author{
H.G. HuGHES \\ Radio Physics Division, U.S. Navy Electronics Laboratory, San Diego, California 92152
}

(Received June 13, 1967)

\begin{abstract}
The mean spectrum of negative polarity 'slow tail' ELF atmospherics received during daytime from the area of a distant mature tropical storm (Hurricane Blanca, August 1966) is presented. The mean amplitude spectrum is used with established attenuation rates to calculate from zero-order mode theory the mean current moment associated with the waveform sources. It is suggested that the waveforms may be greatly controlled by intracloud lightning discharges associated with the degree of vertical development of the hurricane.
\end{abstract}

\section{Introduction}

At extremely low-frequencies (ELF) it is necessary to consider only the zero-order mode of propagation in the earth-ionosphere waveguide to describe the radiated electric fields of distant lightning discharges. Thus, electric field measurements of 'slow tail' atmospherics (Liebermann, 1956) provide a mechanism for studying lightning discharge source characteristics at distances of several thousands of kilometers. This, of course, is possible only if the source distance and propagation parameters of the earth-ionosphere waveguide are known along the path from source to receiver.

During the month of August 1966, simultaneous recordings of 'slow tail' vertical and horizontal electric field components were made in a remote area of the Hawaii Volcanoes National Park, Hawaii. The measurements were made using a vertical antenna and a set of orthogonal earth-probes oriented parallel and perpendicular to the local geomagnetic meridian. The detailed description of the instrumentation and method of source location has been reported elsewhere (Hughes. 1967). The system voltage transfer functions are shown in Figure 1 for the Hawaiian measurements.

Subsequent analysis of the recorded data indicated an area of intense 'sferic' activity located at approximately $\lambda 120^{\circ} \mathrm{W} ; L 22^{\circ} \mathrm{N}$. An examination of synoptic meteorological data indicated that at the times of recording this was the approximate location of a mature tropical storm, Hurricane Blanca. Recordings had been made during the period of local noon on 3, 4 and 5 August 1966 such that the propagation path from the disturbance was under full daytime conditions. A typical example of the 'slow tail' waveforms received from the storm area is shown in Figure 2A. The differences in appearance of the earthprobe signatures and those of the vertical antenna can be attributed mainly to the differences in frequency response of the three systems. There is approximately a $45^{\circ}$ phase difference between the vertical electric field in air and the horizontal electric field in the earth, 


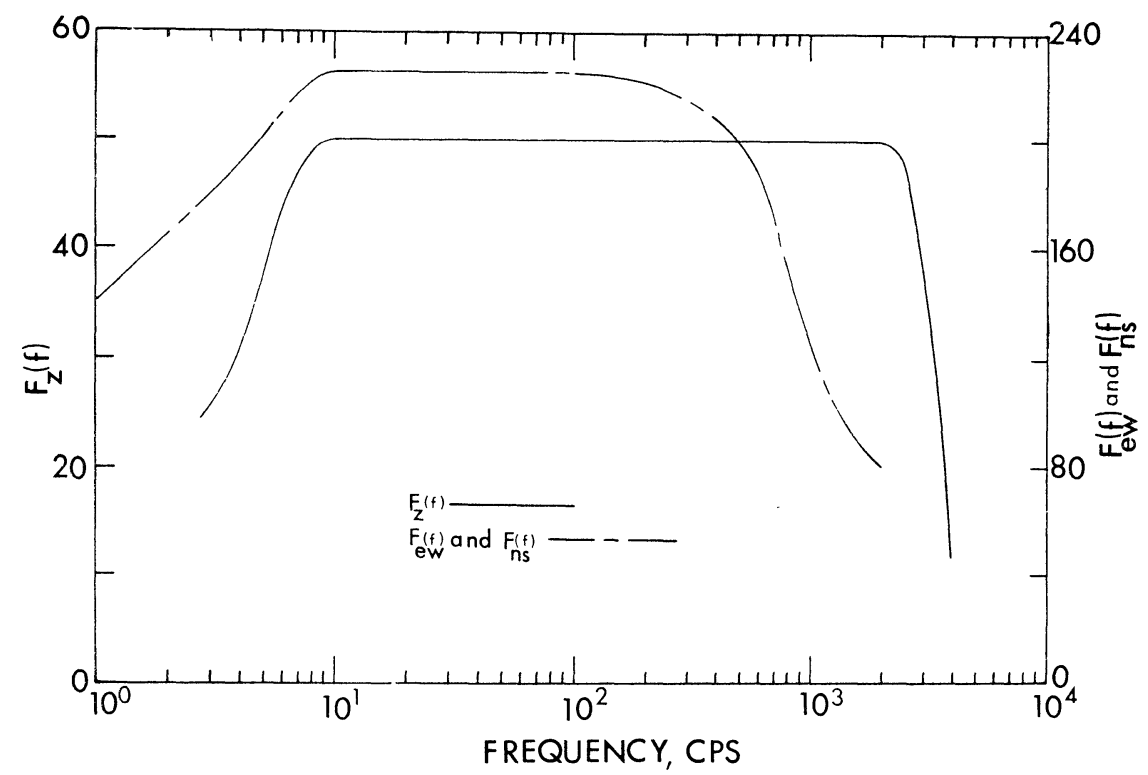

Fig. 1. Voltage transfer functions versus frequency for the vertical antenna system, $F_{Z}(f)$, and for the E-W and N-S earth-probe systems, $F_{e w}(f)$ and $F_{n s}(f)$, restively.

Fig. 2. (A) Example of typical negative 'slow tail' waveforms received from Hurricane Blanca under daytime conditions. The first and third traces in each example are the vertical antenna signature of the same waveform and the second and fourth traces are of the E-W and $\mathrm{N}$-S earth-probe signatures, respectively. In each case, the horizontal scale represents time increasing to the right and each major division corresponds to $2.5 \mathrm{msec}$. The vertical scale is the system output voltage and corresponds to 1.0 volt per major division in each case.

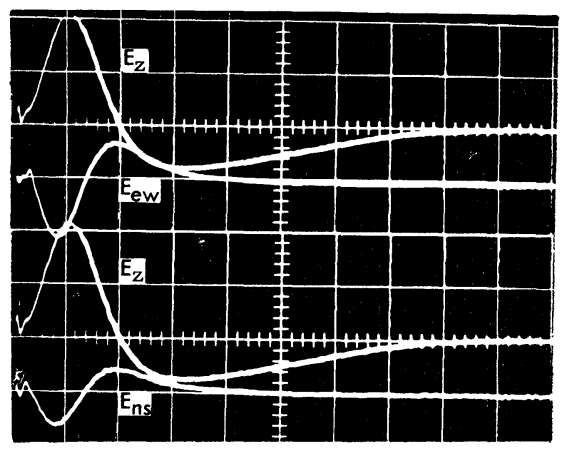

(A)

(B) Approximate locations of the waveform A with respect to Hurricane Blanca.

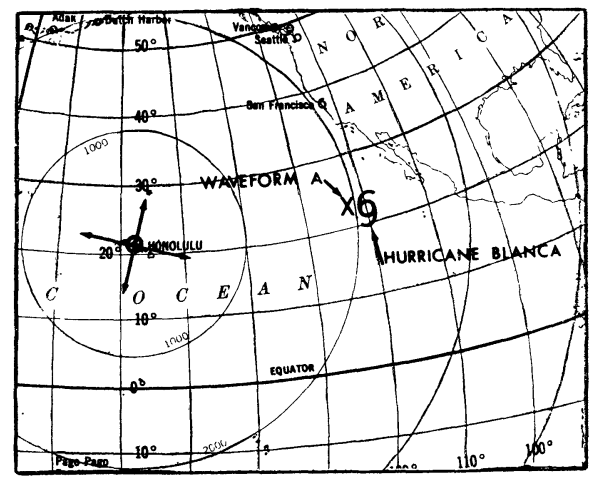


and the ratio of the frequency spectra of the two components is proportional to the square root of the frequency. These two factors account for some of the differences in appearance of the vertical and horizontal waveforms. The approximate location of the received waveform with respect to the reported storm position at 0000Z, 4 August 1966 is shown in Figure 2B. The storm location agrees well with the source distance estimated from the rise time of the vertical antenna signature (Wait, 1962) and the bearing estimated from the ratio of the peak amplitude of the earth-probe signatures. Ideally, the source location should have been shown on an azimuthal-equidistance map with the measurement site at the center. The measurement site on the island of Hawaii was approximately 200 nautical miles east of Honolulu, Oahu.

The vertical antenna signature shown in Figure 2A is of negative polarity. The initial portion of 'slow tail' waveforms may be of either positive or negative polarity (Hepburn, 1957; Tepley, 1961). The term 'negative polarity' is here taken to mean that the electric vector of the initial portion of the waveform is directed downward at the receiving antenna and 'positive polarity' means that the electric vector of the initial portion of the waveform is directed upward. At ELF, the change in phase upon reflection at the boundaries of the earth-ionosphere waveguide is small and the spectral components of the 'slow tail' move down the waveguide in an almost coherent fashion. The initial portion of the waveform has a polarity in the direction of current flow in the lightning discharge and this polarity does not change during propagation (within the distance under consideration) through the waveguide. Because of the dispersive properties of the waveguide only the rise time, amplitude and duration of the pulse change as the distance to the source increases.

Tepley (1961) and Hepburn (1957) have suggested that the 'slow tails' of positive polarity have their origin in the return stroke to cloud of a cloud-ground (CG) discharge and Tepley (1961) has suggested that the 'slow tails' of negative polarity are caused by intracloud (IC) discharges which result from the transfer of positive charge at the top of a cloud to its base. However, an attempt to identify from elementary theory the positive waveforms with CG flashes and the negative waveforms with IC flashes can be misleading. From studies of the 'fine structure' of lightning discharges, Brook and Kitagawa (1960) point out the possibility that the $K$-changes (generated when an advancing junction streamer encounters a region of minor charge concentration) of a lightning discharge contribute considerable energy to the radiated field at ELF. $K$-changes occur for both IC and CG discharges and can be both positive and negative polarity. There is some evidence that the total field change is actually the integrated sum of the individual $K$-changes (Kitagawa and Brook, 1960). However, from the correlations of near field measurements and visual observations of lightning discharges (Bruce and Golde, 1941; Ishikawa, 1961; Müller-Hillebrand, 1963; Pierce, 1955; Smith, 1957) it must be concluded that 5 percent of CG flashes and 20 percent of IC flashes do not follow the elementary rule for the direction of current flow.

During the measurement period, the waveforms received from the storm area were predominately of negative polarity. It is estimated that the number of negative waveforms exceeded the number of positive waveforms by at least one order of magnitude. The number 
of positive waveforms from frontal and convective thunderstorm activity may be expected to be greater than the number of negative waveforms by a factor of 2 or 3 (Hepburn, 1957; Pierce, 1955). It is possible, however, that the data of Hepburn (1957) may have also included waveforms of hurricane origin. A positive statement cannot be made at this time as to the origin of the waveforms analyzed in this paper. However, the degree of vertical development of a hurricane can be great and the intracloud activity can be expected to be intense. It is therefore highly probable that the negative waveforms reported on in this paper are associated with intracloud type lightning discharges.

\section{Data Analysis}

25 waveforms of negative polarity emanating from the area of Hurricane Blanca were analyzed from data recorded around local noon on 4 August 1966. The mean rise time and duration of the sampled daytime waveforms was 2.2 and $21.3 \mathrm{msec}$ with standard deviations of 0.37 and $1.4 \mathrm{msec}$, respectively. The peak amplitudes of the various waveforms were between 3 and $30 \mathrm{mv} / \mathrm{m}$.

The vertical antenna system output voltage, $V(t)$, for each waveform was sampled at a time interval, $\Delta t$, of $0.25 \mathrm{msec}$. The frequency content of each waveform was then determined by numerical evaluation of the complex Fourier transform equation given by

$$
\left.\begin{array}{rl}
E(f) & =\int_{0}^{\infty} \frac{V(t)}{F_{z}(f) l} e^{-i 2 \pi f t} d t \\
& \cong \sum_{n=0}^{\tau / \Delta t}\left[\frac{V(n \Delta t)+V[(n+1) \Delta t]^{-i 2 \pi f\left(n+\frac{1}{2}\right) \Delta t}}{2 F_{z}(f) l}\right] \Delta t .
\end{array}\right\}
$$

$F_{z}(f)$ is the vertical antenna system voltage transfer function, $l$ is the effective half-length

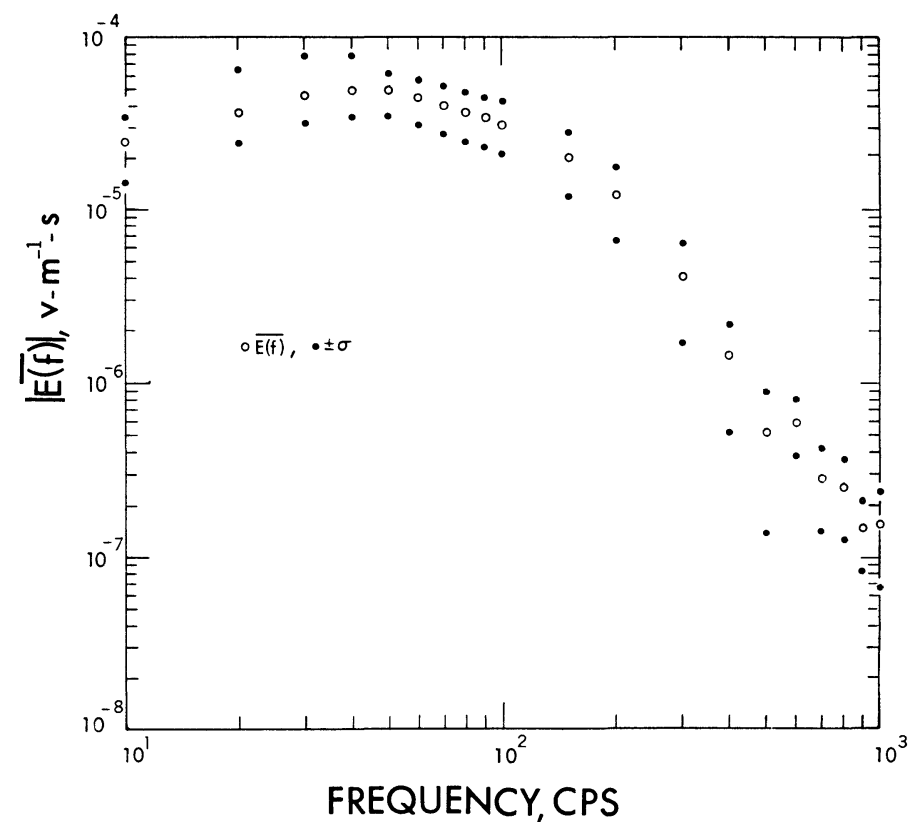

Fig. 3. The mean amplitude spectrum of the waveforms emanating from the area of Hurricane Blanca. 
of the antenna, $\tau$ is the duration of the waveform and $f$ is the frequency. The spectrum of each waveform was then used to synthesize the sampled waveforms by means of the inverse Fourier transform. Truncation errors in sampling the waveforms were found to be small since the synthesized waveforms differed from the sampled waveforms by negligible amounts. Figure 3 shows the mean value $|\overline{E(f)}|$ and standard deviation $\sigma$ of the 25 unsmoothed spectra. The form of the mean spectrum in Figure 3 would be expected from zeroorder mode theory assuming a realistic (electron) ionosphere (Galejs, 1966; Wait, 1962).

\section{Formulation of Current Moment}

The modulus of the frequency spectrum of the received vertical electric field, $|E(f)|$, is related to the modulus of the current moment, $|\operatorname{Ids}(f)|$, by the expression for the zero-order mode (Wait, 1962)

$$
|E(f)|=\frac{\eta \sqrt{f}}{4 \pi h \sqrt{\rho c}}|I d s(f)| \cdot\left|S_{0}(f)\right|^{3 / 2} e^{-\alpha(f) \rho}
$$

where $\eta$ is the impedance of free space, $h$ is the effective height of the ionosphere, $c$ is the free space speed of light, $f$ is the wave frequency, $\rho$ is the distance to the source, $\alpha(f)$ is the attenuation per unit distance and $S_{0}(f)$ is the sine of the complex angle of incidence on the lower ionosphere given by

$$
S_{0}(f) \cong 1-\frac{i^{3 / 2} c}{2^{3 / 2} h \sqrt{\pi f \omega_{r}}}
$$

where $\omega_{r}=\omega_{0}^{2} / v$ in terms of the plasma frequency $\omega_{0}$ and electron collision frequency $v$. The expression for $|E(f)|$ is valid in regions corresponding to the radiation field of the source. From the experimental data of Hepburn (1957), Wait (1962) deduced values of $\omega_{r}$ and $h$ for daytime conditions of $1.2 \times 10^{5} \mathrm{sec}^{-1}$ and $70 \mathrm{~km}$, respectively. Using these values, $\left|S_{0}(f)\right|$ can be determined from equation 3. The mean current moment of the waveform sources is then computed from equation 2 using the values of $|\overline{E(f)}|$ shown in Figure 3. A source distance to Hurricane Blanca of 3500 $\mathrm{km}$ and the measured daytime attenuation rates of Chapman and Macario (1956) were used in the calculations. Figure 4 shows the spectrum of the mean current moment for the frequency range of $100 \mathrm{c} / \mathrm{s}$ to $1000 \mathrm{c} / \mathrm{s}$. Data are not presented below $100 \mathrm{c} / \mathrm{s}$ because this is the lower limit of the Chapman and Macario attenuation

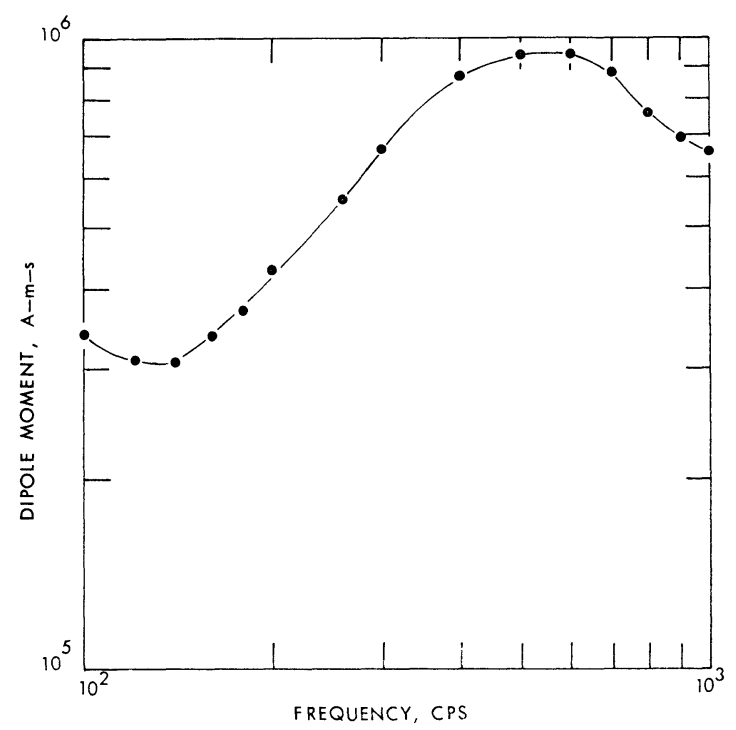

Fig. 4. The mean_current dipole moment versus frequency. 
rates.

\section{Discussion}

Information concerning measurements of lightning discharge current moments below $1 \mathrm{kc} / \mathrm{s}$ is scarce in the published literature. However, assuming free-space radiation, Galejs (1967) has transformed the current of williams (1958) (derived from VLF radiation field measurements) to obtain an expression for the current moment of a median lightning flash (also see Raemer, 1961). Only the waveform signatures of large amplitude lightning discharges are apt to be received above background noise at a large distance from the source, and the current moment of Galejs (1967) is not of sufficient amplitude to explain the waveforms presented here (private communication, Janis Galejs). The current moment of Galejs had to be increased approximately $40 \mathrm{~dB}$ over the frequency range from $100 \mathrm{c} / \mathrm{s}$ to $1 \mathrm{kc} / \mathrm{s}$ to reasonably predict the measured field strengths using the attenuation rates of Chapman and Macario (1956). At a frequency of $10 \mathrm{c} / \mathrm{s}$ the calculated current moment of Galejs is approximately $20 \mathrm{~dB}$ lower than the mean current moment determined by Rycroft (1965) from electric field measurements of the earth-ionosphere cavity resonances. Also, at VLF the radiation fields calculated by Galejs using his mean current moment were approximately $26 d B$ lower than the radiation field measurements of Taylor (1960).

The current moment shown in Figure 4 cannot be interpreted as being representative of the median of all lightning flashes, but only as the mean current moment responsible for the waveforms analyzed here. The waveforms analyzed here may be associated with intracloud discharges in Hurricane Blanca; and the large values of the current moment in Figure 4 may possibly be due to the exceptionally long discharge channel length associated with the vertical development of the storm. The present technique of source location does not allow sufficient range resolution to determine if the waveforms emanated from the storm periphery or the regions of intense winds. However, the waveforms were received during the mature stage of the hurricane.

\section{References}

Bruce, C.E.R. and R.H. Golde, The lightning discharge, J. Inst. Elec. Engrs. (London), 88 487-505, 1941.

Brook, M. and Kitagawa, Electric field changes and the design of lightning-flash counters, J. Geophys. Res., 65, 1927-1931, 1960.

Chapman, F.W., and R.C.V. Macario, Propagation of audio-frequency radio waves to great distances, Nature, 177, 930-933, 1956.

Galejs, J., Amplitude distributions of radio noise at ELF and VLF, J. Geophys. Res., 71 (1), 201-216, 1966.

Galejs, J., Amplitude statistics of lightning discharge currents and ELF and VLF radio noise, J. Geophys. Res., to appear 1 June 1967.

Hepburn, F., Atmospheric waveforms with very low frequency components below $1 \mathrm{kc} / \mathrm{s}$ known as slow tails, J. Atmosph. Terrest. Phys., 10, 266-287, 1957.

Hughes, H.G., On the directional dependency of 'slow tail' extremely low-frequency atmospheric waveforms, J. Atmosph. Terrest. Phys., to appear 1967.

Ishikawa, H., Nature of lightning discharges as origins of atmospherics, Proc. Res. Inst. Atmospherics, Nagoya Universisy, 8A, 1-274, 1960. 
Kitagawa, N. and M. Brook, A comparison of intracloud and cloud-to-ground lightning discharges, $J$. Geophys. Res., 65, 1159-1201, 1960.

Liebermann, L., Extremely low-frequency electromagnetic waves : I. Reception from lightning, J. Appl. Phys., 27, 1473-1476, 1956.

Müller-Hillebrand, D., Lightning Counters-II. The effect of changes of electric field on counter circuits, Arkiv. Geof. 4, 271-292, 1963.

Pierce, E.T., Electrostatic field-changes due to lightning discharges, Quart. J. Roy. Meteoral. Soc., 81, 211-228, 1955.

Raemer, H.R., On the spectrum of terrestrial radio noise at ELF, J. Res. NBS(Radio Propagation), 65D (6), 581-594, 1961.

Rycroft, M.J., Resonances of the earth-ionosphere cavity observed at Cambridge, England, Radio Science, 69, 1071-1081, 1965.

Smith, L.G., Intracloud lightning discharges, Quart. J. Roy. Meteoral. Soc., 83, 103-111, 1957.

Taylor, W.L., Daytime attenuation rates in the VLF band using atmospherics, J. Res. NBS (Radio Propagation), 64D (4), 349-355, 1960.

Tepley, L.R., Sferics from intracloud lightning strokes, J. Geophys. Res., 66, 111-123, 1961.

Wait, J.R., Electromagnetic Waves in stratified media, The Macmillan Company, New York, 1962.

Williams, J.C., Thunderstorms and VLF radio noise, Ph.D. thesis, Harvard University, May 1959. 